\title{
Ultrasound and Elastographic Features predicting Axillary Nodal Metastasis in Breast Cancer
}

\author{
${ }^{1}$ Suresh V Phatak, ${ }^{2}$ Raju K Shinde, ${ }^{3}$ Nipun A Gupta, ${ }^{4}$ Gaurangi Pawar, ${ }^{5}$ Pallavi S Phatak
}

\begin{abstract}
Lymph node metastasis is an important prognostic factor in cases of carcinoma breast. In this pictorial essay, various sonographic and elastographic signs of lymph node metastasis are described with classical images.
\end{abstract}

Keywords: Axilla, Breast, Elastography, Metastasis, Ultrasound.

How to cite this article: Phatak SV, Shinde RK, Gupta NA, Pawar G, Phatak PS. Ultrasound and Elastographic Features predicting Axillary Nodal Metastasis in Breast Cancer. Int J Recent Surg Med Sci 2017;3(2):127-129.

Source of support: Nil

Conflict of interest: None

\section{INTRODUCTION}

Axillary lymph node metastasis is an important prognostic factor in early-stage breast cancer and remains crucial on which individual treatment decisions are made. ${ }^{1}$ Mammography has its limitations in detecting metastatic lymph nodes because the entire axillary region cannot be adequately evaluated with this method. Positron emission tomography combined with computed tomography and magnetic resonance imaging do not have a place in routine staging because of higher costs and possibility of side effects. ${ }^{2}$ Ultrasonography (USG) is the most widely used imaging method for detection of axillary lymph node metastasis and for their characterization. $^{3}$

\footnotetext{
${ }^{1,2}$ Professor and Head, ${ }^{3,4}$ Resident, ${ }^{5}$ Student (Final Year)

1,3,4 Department of Radiodiagnosis, Jawaharlal Nehru Medical College, Wardha, Maharashtra, India

${ }^{2}$ Department of General Surgery, Jawaharlal Nehru Medical College Wardha, Maharashtra, India

${ }^{5}$ Jawaharlal Nehru Medical College, Wardha, Maharashtra, India

Corresponding Author: Suresh $\mathrm{V}$ Phatak, Professor and Head, Department of Radiodiagnosis, Jawaharlal Nehru Medical College, Wardha, Maharashtra, India, Phone: +917152287701 e-mail: suresh_phatak@yahoo.com
}

\author{
Ultrasonography Appearance of Normal Axillary \\ Lymph Node (Fig. 1)
}

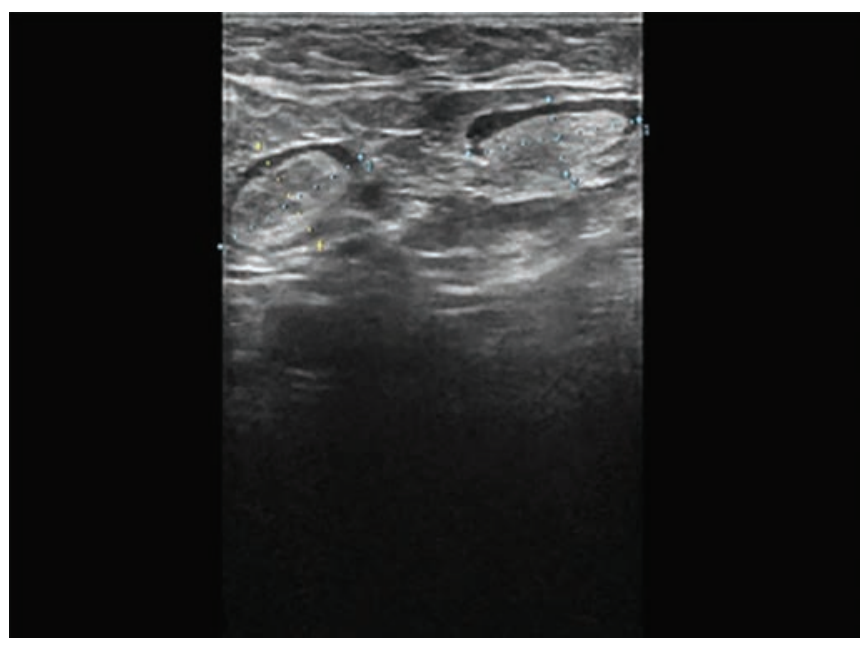

Fig. 1: Normal axillary node is seen as elongated morphology, with an elliptical shape, containing a high proportion of central echogenic tissue, due to fat, network of blood and lymphatic vessels in the hilum. The cortex is hypoechoic, with its echogenicity similar to that of the hypoechoic subcutaneous fat

\section{Ultrasonography Features of Malignant Axillary Lymph Node}

\section{Cortical Thickening (Fig. 2)}

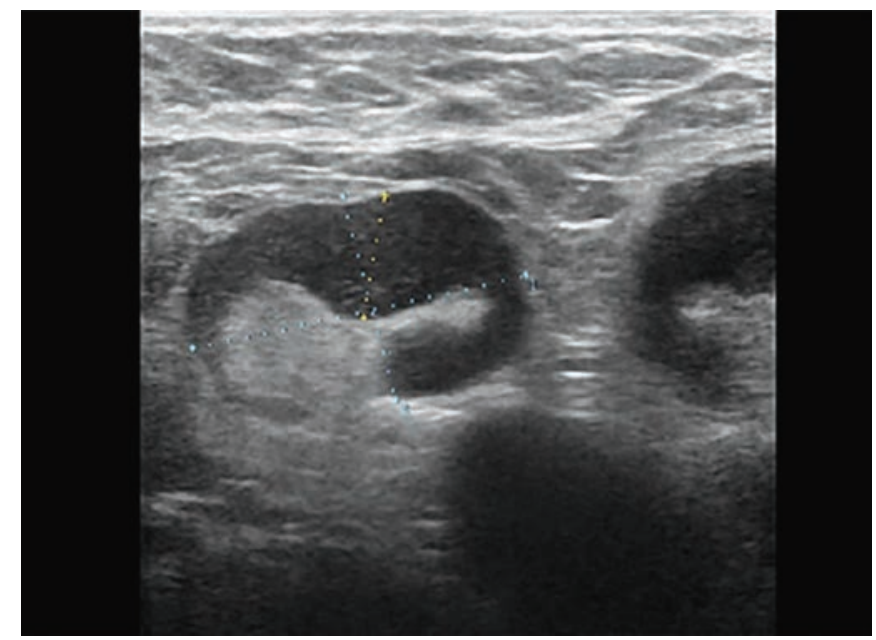

Fig. 2: Eccentric cortical thickening is seen here, which is caused by metastasis arrested in periphery of node. This usually happens before generalized cortical enlargement 


\section{Marked Hypoechogenicity and Loss of Fatty Hilum (Fig. 3)}

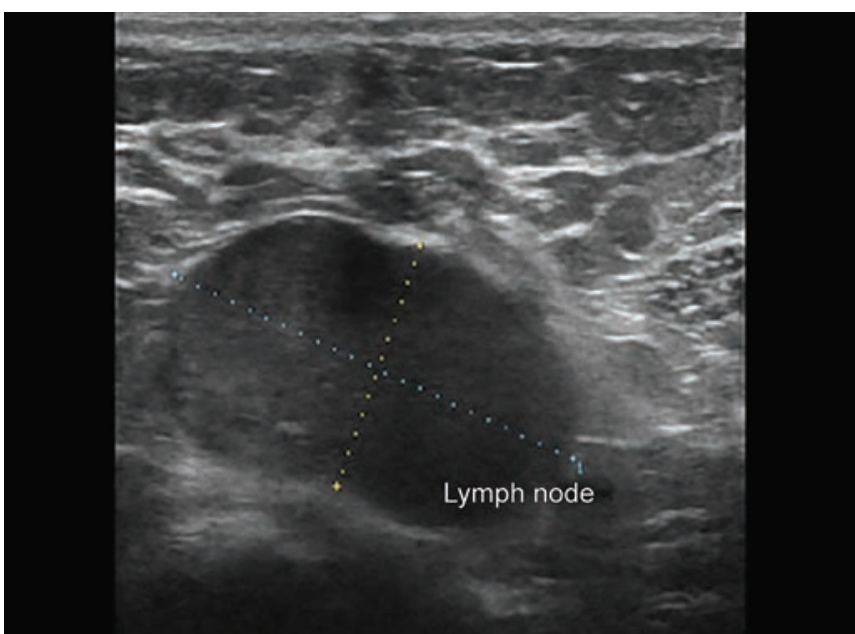

Fig. 3: Marked hypoechogenicity and a fatty hilum loss attributable to metastatic deposits in lymph node

\section{Calcification (Fig. 4)}

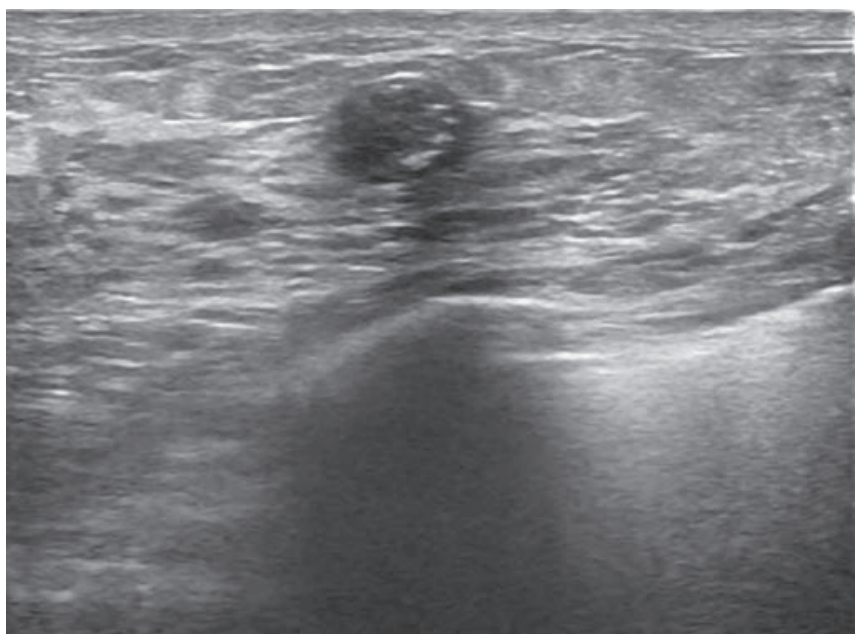

Fig. 4: Hypoechoic axillary node containing calcification similar to primary tumor in breast is hallmark of metastasis

\section{Capsular Breach (Fig. 5)}

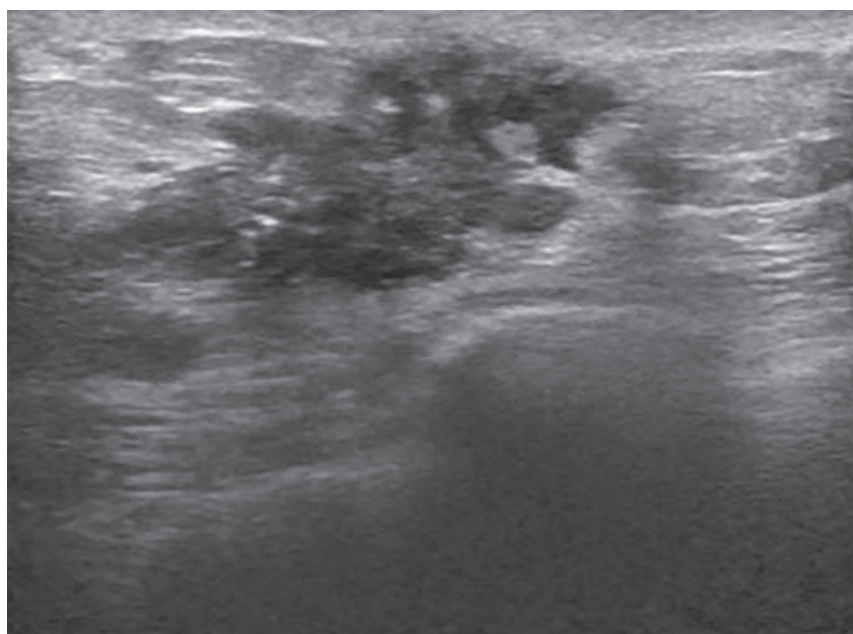

Fig. 5: Capsular breach in a lymph node is a salient feature of metastatic lymph node

\section{Longitudinal-transverse Axis Ratio (Fig. 6)}

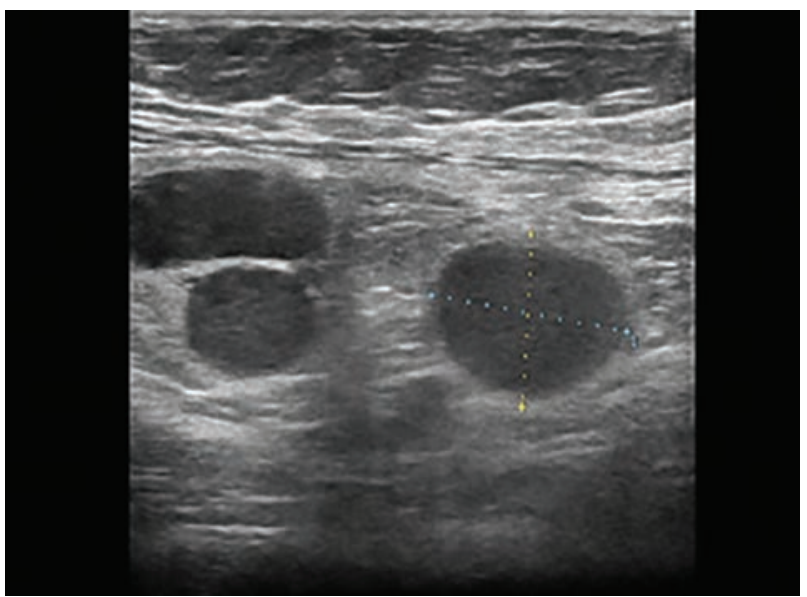

Fig. 6: Enlargement of the short plane of the lymph node causing low longitudinal-transverse axis ratio (1.1; longitudinal short-axis ratio less than 2 is significant), due to the neoplastic involvement enlarging the short plane of the lymph node causing round shape of node, which is seen in malignant nodes

\section{Cortex to Hilum Ratio (Figs 7 and 8)}

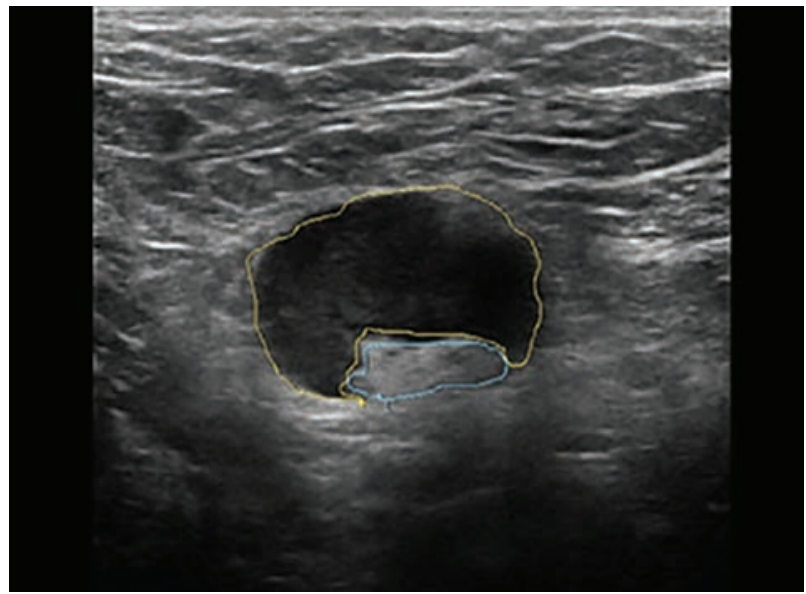

Fig. 7: Metastatic lymph node showing cortex to hilum of 2.6 taken by trace method. A value more than 2 is an important feature of metastatic lymph node. Hilum is displaced at the periphery of node

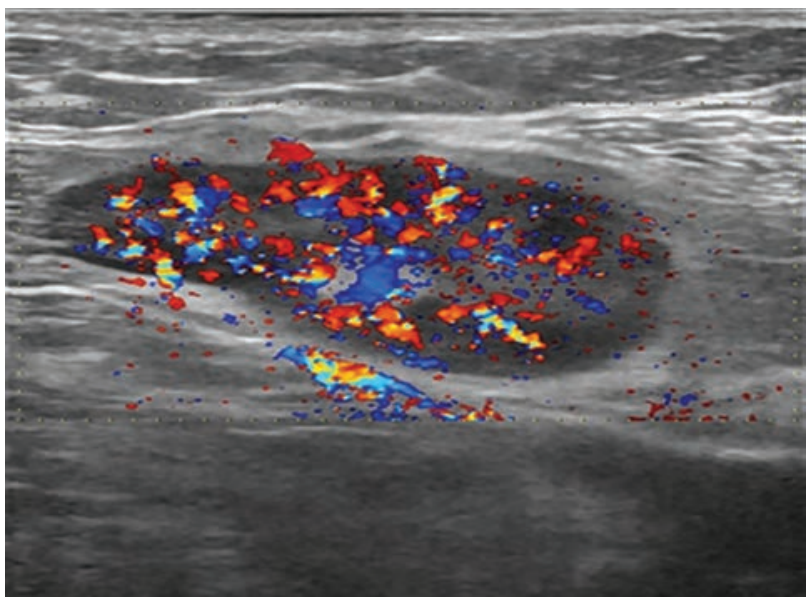

Fig. 8: Color Doppler showing peripheral blood flow in subcapsular region due to tumor angiogenesis is also a characteristic of metastatic lymph node 


\section{Sonoelastographic Findings in Metastatic Lymph Node (Figs 9 and 10)}

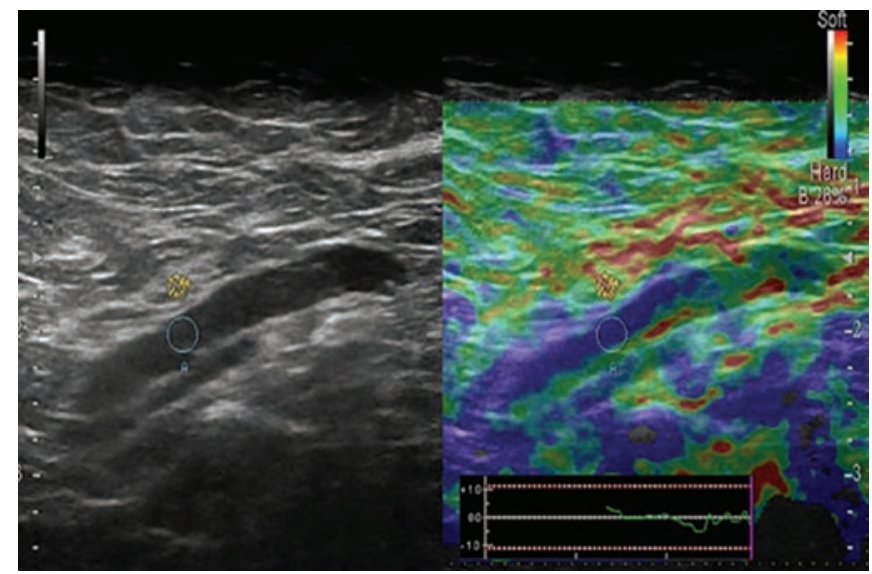

Fig. 9: Although elliptical, this lymph node is showing eccentric cortical thickening, and on elastography stiff color is noted in cortex (blue), indicating metastatic deposit confirmed on fine needle aspiration cytology. Strain ratio of lymph node in this case was 61.67

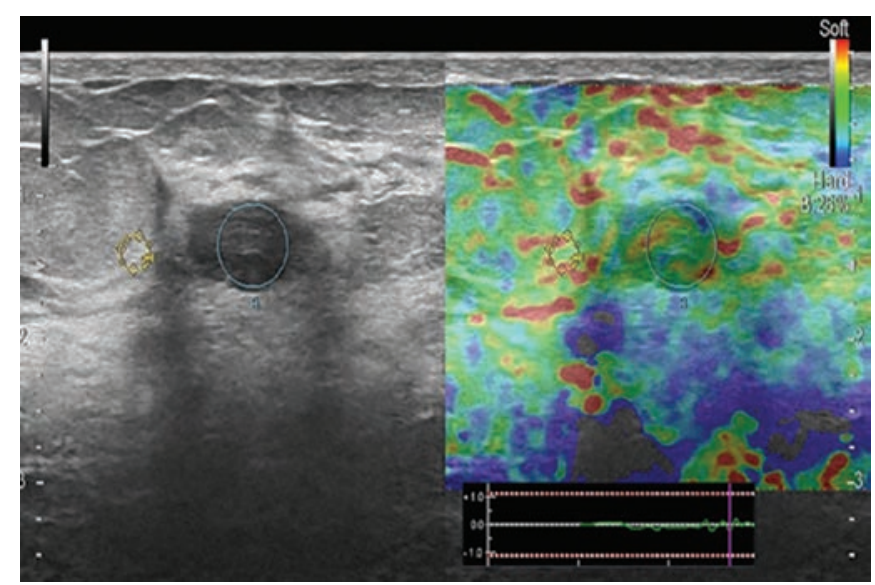

Fig. 10: Reactive lymph node in this case of carcinoma breast showing soft colors (score 1 ) with a strain ratio of 1.59 , indicating benign nature of disease

\section{Elastography Scoring System for Metastatic Lymph Node (Fig. 11)}

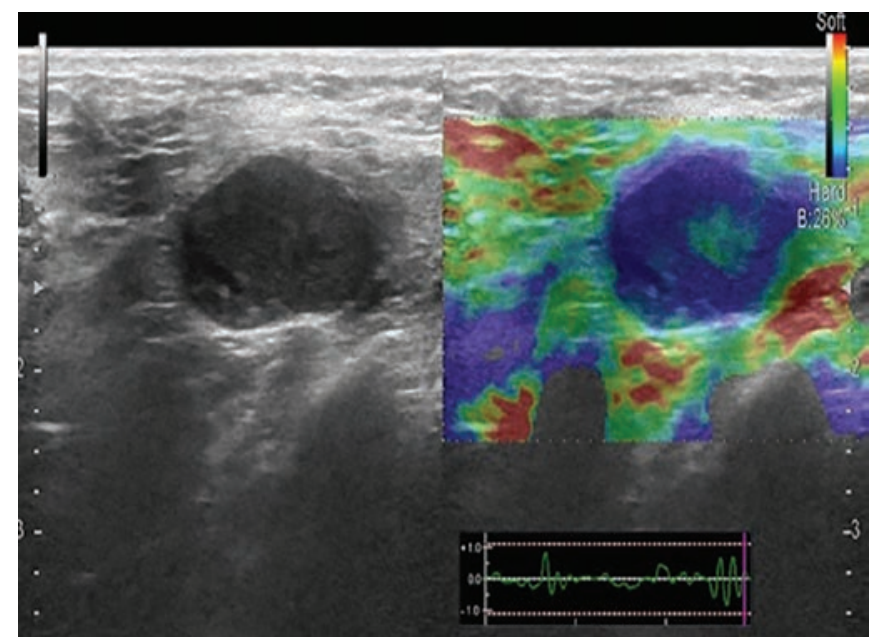

Fig. 11: Metastatic lymph node showing score of 4 on elastography score system (blue area more than $45 \%$ with or without green rim)

\section{SUMMARY}

Metastasis to axillary lymph node is an important factor in the prognosis of early-stage breast cancer and crucial for decision-making in management. There are many classical gray-scale and elastographic features that predict metastasis to axillary lymph node. These features are discussed in brief and classical images are illustrated in patients of breast cancer. These features help in early detection of lymph node metastasis.

\section{REFERENCES}

1. Stachs A, Tra-Ha Thi A, Dieterich M, Stubert J, Hartmann S, Glass Ä, Reimer T, Gerber B. Assessment of ultrasound features predicting axillary nodal metastasis in breast cancer: the impact of cortical thickness. Ultrasound Int Open 2015 Jul;1(1):E19-E24.

2. Peare R, Staff RT, Heys SD. The use of FDG-PET in assessing axillary lymph node status in breast cancer: a systematic review and meta-analysis of literature. Breast Cancer Res Treat 2010 Aug;123(1):281-290.

3. Houssami N, Ciatto S, Turner RM, Cody HS 3rd, Macaskill P. Preoperative ultrasound-guided needle biopsy of axillary nodes in invasive breast cancer: meta-analysis of its accuracy and utility in staging the axilla. Ann Surg 2011 Aug;254(2):243-251.

4. Fornage BD. Local and regional staging of invasive breast cancer with sonography: 25 years of Practice at MD Anderson Cancer Center. Oncologist 2014 Jan;19(1):5-15.

5. Whitman GJ, Lu TJ, Adejolu M, Krishnamurthy S, Sheppard D. Lymph node sonography. Ultrasound Clin 2011 Jul;6:369-380.

6. Feu J, Tresserra F, Fábregas R, Navarro B, Grases PJ, Suris JC, Fernández-Cíd A, Alegret X. Metastatic breast carcinoma in axillary lymph nodes: in vitro US detection. Radiology 1997 Dec;205(3):831-835.

7. Song SE, Seo BK, Lee SH, Yie A, Lee KY, Cho KR, Woo OH, $\mathrm{Cha} \mathrm{SH}, \mathrm{Kim} \mathrm{BH}$. Classification of metastatic versus nonmetastatic axillary nodes in breast cancer patients: value of cortex-hilum area ratio with ultrasound. J Breast Cancer 2012 Mar;15(1):65-70.

8. Wojcinski S, Dupont J, Schmidt W, Cassel M, Hillemanns P. Real-time ultrasound elastography in 180 axillary lymph nodes: elasticity distribution in healthy lymph nodes and prediction of breast cancer metastases. BMC Med Imaging 2012 Dec;12:35.

9. Choi JJ, Kang BJ, Kim SH, Lee JH, Jeong SH, Yim HW, Song BJ, Jung SS. Role of sonographic elastography in the differential diagnosis of axillary lymph nodes in breast cancer. J Ultrasound Med 2011 Apr;30(4):429-436. 